\title{
Control interno y las contrataciones inferiores a 8 UIT en la UGEL Vilcas Huamán, Ayacucho 2021
}

\author{
Mg. CPC Manuel Jesús García Amaya \\ cpcmajesu_23@hotmail.com \\ ORCID: 0000-0001-6369-8627 \\ Universidad Nacional \\ San Luis Gonzaga \\ Ica - Perú \\ whatsapp: 966621544
}

\section{CPC. Walter Prado Alarcón}

walterpradoa@gmail.com ORCID: 0000-0002-6993-1095

Universidad Privada

Católica Los Ángeles de Chimbote

Ayacucho - Perú

\section{RESUMEN}

La investigación fue buscar la relación entre el control interno y las contrataciones inferiores a 8 UIT en la UGEL Vilcas Huamán, Ayacucho 2021, planteándose la metodología con un enfoque cuantitativo, tipo básica y nivel correlacional, diseño no experimental, con una muestra de 43 servidores, se utilizó la técnica de encuesta y recolección de datos el cuestionario. Llegando, a las conclusiones siguientes, el control interno y las contrataciones inferiores a 8 UIT tienen relación positiva con un resultado de $(r=0.565)$. Igualmente, se determinó que existe relación entre el ambiente del control interno y las contrataciones inferiores a 8 UIT, siendo positivo $(r=0.373)$. Asimismo, se evidencio que gestión de riesgo y las contrataciones inferiores a 8 UIT se relacionan, obteniendo un resultado significativo de $(\mathrm{r}=0,584)$. De la misma forma, se describió que las actividades de control se relacionan con las contrataciones inferiores a 8 UIT, siendo positiva ( $\mathrm{r}=0.402)$. También, se estableció que la información y comunicación se relaciona con las contrataciones inferiores a 8 UIT con un resultado positivo de $(\mathrm{r}=0.591)$ y, por último, se observó que la supervisión se relaciona con las contrataciones inferiores a 8 UIT con un resultado positivo de $(\mathrm{r}=0.517)$.

Palabra clave: control interno; contrataciones; unidad de gestión educativa local 


\title{
Internal control and hiring of less than 8 UIT in the UGEL Vilcas Huamán, Ayacucho 2021
}

\begin{abstract}
The research was to seek the relationship between internal control and hiring of less than 8 UIT in the UGEL Vilcas Huamán, Ayacucho 2021, considering the methodology with a quantitative approach, basic type and correlational level, non-experimental design, with a sample of 43 servers The survey technique and the questionnaire data collection were used. Reaching the following conclusions, internal control and hiring of less than 8 UIT have a positive relationship with a result of $(\mathrm{r}=0.565)$. Likewise, it was determined that there is a relationship between the internal control environment and hiring of less than 8 UIT, being positive $(r=0.373)$. Likewise, it was evidenced that risk management and contracts of less than 8 UIT are related, obtaining a significant result of $(r=0.584)$. In the same way, it was described that control activities are related to hiring of less than 8 UIT, being positive $(r=0.402)$. Also, it was established that information and communication is related to hiring of less than 8 UIT with a positive result of $(r=0.591)$ and, finally, it was observed that supervision is related to hiring of less than 8 UIT with a result positive of $(r=0.517)$.
\end{abstract}

Keyword: internal control; hiring; local educational management unit

Artículo recibido: 15 enero 2022 Aceptado para publicación: 08 febrero 2022 Correspondencia: cpcmajesu_23@hotmail.com Conflictos de Interés: Ninguna que declarar 


\section{INTRODUCCIÓN}

En el ámbito mundial la corrupción en el mundo según PROETICA (2020) indica que los movimientos anticorrupción del mundo recibieron apoyo de miles de ciudadanos que se unieron para protestar contra el cáncer de la corrupción que viene atravesando sus países. Las manifestaciones en los países de Sudamérica, África, Europa, Oriente Medio y Asia Central recogieron la atención de la prensa. Los ciudadanos están cansados de la corrupción que ocurre desde los altos mandos del gobierno hasta los sobornos que obstaculizan el acceso a los servicios estatales de la enseñanza y salud. Asimismo, establece que, para bajar los actos de corrupción, los países deben de fortalecer para mitigar la corrupción, los estados deben fortalecer y proteger los sistemas de control.

También, a nivel internacional, según Miaja Fol (2019) finalizó que el control interno en el sistema público de España se encuentra en dificultades por el resultado de su incapacidad para advertir una pésima gestión, el fraude y la corrupción. Consecuencia de continuos cambios en la forma de realizar el control. Mientras, que, en la región de Colombia, Montiel et al (2017) estableció que a través del Modelo Estándar de Control Interno - MECI, proporciona procesos para dirigir, comunicar y realizar el autocontrol y supervisión de las acciones diarias a cada integrante de la organización, asimismo, es importante el compromiso de la más alta Dirección en realizar cambios que fortalezcan y mejoren la Entidad para cumplir los objetivos.

En el nivel nacional el Organismo Supervisor de las Contrataciones del Estado se dictaminaron diversas reglas como la Ley de Contrataciones del Estado, aprobada mediante la Ley $\mathrm{N}^{\circ}$ 30225, modificada por los Decretos Legislativos $1341 \mathrm{del} 7$ de enero del 2017 y 1444 de 16 de setiembre de 2018. Finalmente, mediante el Decreto Supremo $\mathrm{N}^{\circ}$ 082-2018-EF de 13 de marzo de 2019 apruebo el TÚO de la Ley de Contrataciones, cuya finalidad es regular que los fondos del estado sean manejados de manera oportuna en las adquisiciones de bienes, servicios y obras, bajo excelente propuesta de costo y calidad, con la finalidad de cumplir los objetivos institucionales y tengan un impacto efectivo en la ciudadanía del Perú. Además, es preciso mencionar que, en unos de sus artículos, expresa que las contracciones inferiores a 8 UIT se encuentran excluidas del entorno de la Ley; sin embargo, deben cumplir con los principios de Libertad de concurrencia, igualdad de trato, transparencia, publicidad, competencia, eficacia y eficiencia, vigencia tecnológica, sostenibilidad ambiental y social, y por último equidad 
encuadrados en la mencionada Ley. Ante lo descrito, las entidades del Estado peruano, vienen adquiriendo bienes y servicios bajo los supuestos excluidos, mediante sus directivas internas, regulando los procedimientos de dicha contratación; sin embargo, no son supervisadas al $100 \%$ por el ente regulador, originando que las contrataciones tengan una gran brecha de riesgos que no se realicen de manera adecuada por la falta de control. Mientras, Arribasplata Sánchez (2018) expresó que los riesgos que encontraron en las contrataciones menores a ocho (8) UIT, fueron fraccionamiento, incumplimiento de los principios y la falta de procedimientos para castigar a los proveedores que incumplen el cronograma de entrega de los productos. Igualmente, Azalgara Bedoya (2019) advirtió la existencia de riesgo de corrupción en las compras menores a 8 UIT, identificado riesgo de direccionamiento y proformas sobre valorizadas, esto se debe por el bajo de nivel de control. Para bajar el grado de corrupción se debe implementar y fortalecer las tecnologías de información en las contrataciones, mejorar las normas legales y buscar nuevas estrategias de control en las adquisiciones, y por último buscar mecanismos de solución de problemas más eficientes y la implementación de procedimientos modernos. De igual manera la Contraloría General de la República (CGR, 2021) señaló que los resultados sobre la corrupción en el Perú entre el 2016 y 2017 se ubicó en el 50\% de los países con los peores puntajes de índice de percepción de corrupción.

Por otro lado, a nivel local se encuentra la Provincia de Vilcas Huamán, donde está ubicado la Unidad de Gestión Educativa Local del mismo nombre, cuya misión es promover ofertar educativas de calidad y de formación integral de los estudiantes, enmarcado en un enfoque de equidad, la UGEL Vilcas Huamán, obtiene un gran presupuesto para bienes y servicios para el 2021; realizando contrataciones inferiores a 8 UIT ya que está debidamente permitido por Ley, con el propósito que la UGEL cumpla con sus objetivos y metas oportunamente. Sin embargo, dichas contrataciones no son evaluadas de manera exhaustiva en el antes y después, llevándose a hechos de presuntas irregularidades en el proceso de la contratación. Asimismo, los requerimientos de las contrataciones son direccionadas a un solo proveedor, los bienes son recepcionados no acorde a las especificaciones técnicas. En cuanto al control interno no está implementado, existiendo un riesgo potencial que la entidad no cumpla con sus objetivos y metas señalas en sus documentos de gestión. 
El planteamiento del problema general ¿Cuál es la relación entre el control interno y las contrataciones inferiores a 8 UIT en la UGEL Vilcas Huamán, Ayacucho 2021 ?

El estudio se justifica porque se va producir nuevos conocimientos en el control interno y las contrataciones inferiores a 8 UIT, estos resultados nos permitirán contar con evidencia para perfeccionar el control interno y los procesos de contrataciones que se lleven con transparencia, economía, eficiencia y eficacia. Los principales beneficiarios, serían las entidades públicas y por ende la UGEL Vilcas Huamán de tal manera se explica lo siguiente: Según, Hernández et. al, (2014) dice que en la justificación se expresa las razones porque es importante el estudio.

En cuanto a la justificación metodológica, se llevará a cabo mediante un proceso estructurado, el cual será de enfoque cuantitativo, de nivel correlacional entre las dos variables de Control Interno y las Contrataciones inferiores a 8 UIT las que están vinculadas entre sí, por ello es necesario aplicar el método científico. Igualmente, la justificación Teórica, ayudara a identificar las dificultades que tiene la UGEL Vilcas Huamán, basada en la aseveración teórica que permite examinar la relación entre las variables del trabajo de investigación, lo que permitirá observar la problemática que tiene la UGEL Vilcas Huamán. Se utilizará la técnica de la encuesta como herramienta de medición permitiendo ver las carencias que se tiene que mejorar. Asimismo, la presente investigación servirá para otras futuras investigaciones similares en lo posterior. Asimismo, la justificación práctica, permitirá resolver los problemas de la investigación del Control y su relación con las Contrataciones menores a 8 UIT. En cuanto a la justificación social, permitirá que la UGEL Vilcas Huamán pueda realizar mejoras en los sistemas administrativos de la ejecución de las compras directas que contienen ciertas irregularidades, advirtiéndose presuntas irregularidades en el proceso de la contratación.

De la misma manera, se planteó el objetivo general: Determinar la relación entre el control interno y las contrataciones inferiores a 8 UIT en la UGEL Vilcas Huamán, Ayacucho 2021.También se planteó la hipótesis general: Existe relación entre el control interno y las contrataciones inferiores a 8 UIT en la UGEL Vilcas Huamán, Ayacucho 2021.

\section{ESTRATEGIAS METODOLÓGICAS O MATERIALES Y MÉTODOS}

El tipo de investigación es básica porque se investiga conocer y extender los conocimientos de la realidad. De acuerdo a Carrasco (2017) advirtió que no tiene 
propósitos inmediatos, porque solo busca extender y ahondar el caudal de instrucciones científicas existentes acerca de la realidad. (p.43)

Asimismo, la investigación se llevó a cabo mediante un enfoque cuantitativo, porque se cuantificará la variable del estudio a nivel de categorías que medirán las dimensiones e indicadores del control interno y contrataciones inferiores a 8 UIT, hecho que concuerda con Hernández et al. (2014) donde señaló que se toma la información para probar la hipótesis mediante las dimensiones e indicadores, además se considera como investigación básica por que indaga temas reales basadas en conocimiento teórico científico del estudio, tal como lo afirma Valderrama (2019) que es el desarrollo de una teoría, basadas en hechos, principios y normas. Mientras Carrasco (2017) expresó que solo busca expandir el conocimiento existente acerca de la realidad.

El diseño es no experimental, porque no se manipulará las variables de estudio, ya que los hechos ocurrieron anterior a la investigación, hecho que coincide con Valderrama (2019) donde explicó que se estudia los hechos reales después de su ocurrencia (p.71). Asimismo, se trata de una investigación de nivel trasversal correlacional ya que asocian, predicen, cuantifican la relación entre conceptos o variables, Carrasco (2017) estableció que busca determinar el grado de relación entre las variables que se investiga.

La población del estudio estuvo compuesta por 48 empleados públicos de la Sede Central de la UGEL Vilcas Huamán. De acuerdo a Carrasco (2019) dijo que la población, es la masa de todos los elementos, seres que corresponden al entorno espacial donde se lleva el estudio (pp. 236-237).

La muestra, es de 43 servidores del total de la población, incluido funcionarios y servidores de la UGEL Vilcas Huamán. Es una parte que se extrae de una población representativa para un determinado estudio (Valderrama, 2017).

Técnica de encuesta que presumirá estimular reacciones sobre conjuntos de preguntas ordenadas vía la aplicación del cuestionario para calcular a los sujetos de análisis de los trabajadores de la UGEL Vilcas Huamán. Es decir, ayuda a recopilar los datos mediante preguntas formuladas a los trabajadores de la muestra (Carrasco, 2017, p. 314).

Para la encuesta se aplicará utilizando como herramienta el cuestionario, teniendo como informantes a los empleados de la UGEL Vilcas Huamán. El instrumento, también será validado en contenido por Calandria, será usado para lograr datos de los dominios ya citados de modo suplementario para cruzar información procedente de los informantes 
trabajadores. Según, Carrasco (2017) el cuestionario es respondido por una gran masa de personas de manera directa y se elabora de acuerdo a las variables, dimensiones e indicadores (pp.318-319).

La validez del cuestionario, se determinó mediante la validación de jueces expertos, señalando que existe relación entre los objetivos plasmados, dimensiones, indicadores, considerando coherencia de los mismos. Lo mencionado tiene relación con Hernández et. al, (2014) donde señala que es el valor en que un instrumento en realidad calcula la variable que se busca medir (p.200). Para determinar la confiabilidad del instrumento, se procedió a llenar los datos en el programa Excel de acuerdo a la encuesta piloto, datos que fueron calculados a mediante el Alfa de Cronbach. De acuerdo al cuestionario del Control interno y las contrataciones inferiores a 8 UIT de la UGEL Vilcas Huamán, el coeficiente de confiabilidad es de 0.74 Según Hernández et. al, (2014) es el valor en que una herramienta origina consecuencias sólidos y coherentes. (p.200).

La confiabilidad se realizó con la fórmula alfa de Cronbach en el programa Excel. Se tomó una muestra de 6 individuos que no se encontraran en la muestra oficial, ellos contestaron cada pregunta de manera ordena.

\section{RESULTADOS Y DISCUSIÓN}

Tabla 1 Pruebas de correlación de las variables control interno y las contrataciones inferiores a 8 UIT en la UGEL Vilcas Huamán, Ayacucho 2021.

\begin{tabular}{llcc}
\hline & Detalle & $\begin{array}{c}\text { Puntaje total del } \\
\text { Control Interno }\end{array}$ & $\begin{array}{c}\text { Puntaje total de la } \\
\text { Contrataciones } \\
\text { inferiores a 8 UIT }\end{array}$ \\
\hline $\begin{array}{l}\text { Puntaje total } \\
\text { Control Interno }\end{array}$ & $\begin{array}{c}\text { del } \\
\text { Correlación de Pearson }\end{array}$ & 1 & $.565^{* *}$ \\
& Sig. (bilateral) & & .000 \\
\hline $\begin{array}{l}\text { Puntaje total de } \\
\text { Contrataciones }\end{array}$ & la Correlación de Pearson & $.565^{* *}$ & 43 \\
inferiores a 8 UIT & Sig. (bilateral) & .000 & 1 \\
\hline
\end{tabular}

**. La correlación es significativa al nivel 0,01 (bilateral).

Nota: El r de Pearson es de 0.565, cuyo valor del Sig, (bilateral) de 0.000, que se encuentra por debajo del 0.01 requerido, por lo cual se refuta la Ho y se acepta la Ha además esta correlación es positiva muy significativa, por lo que se puede afirmar con un $99 \%$ de confianza, que el ámbito de estudio entre el control interno y las contrataciones inferiores a 8 UIT en la UGEL Vilcas Huamán, Ayacucho 2021 existe una relación positiva considerable $(\mathrm{r}=0.565)$. 


\section{Tabla 2}

Pruebas de correlación de la dimensión ambiente de control interno y la variable de las contrataciones inferiores a 8 UIT en la UGEL Vilcas Huamán, Ayacucho 2021.

\begin{tabular}{|c|c|c|c|}
\hline \multicolumn{2}{|c|}{ 2Detalle } & $\begin{array}{l}\text { Puntaje total de la } \\
\text { Contrataciones } \\
\text { inferiores a } 8 \text { UIT }\end{array}$ & $\begin{array}{l}\text { Ambiente de } \\
\text { control interno }\end{array}$ \\
\hline \multirow{3}{*}{$\begin{array}{l}\text { Puntaje total de la } \\
\text { Contrataciones inferiores } \\
\text { a } 8 \text { UIT }\end{array}$} & Correlación de Pearson & 1 & $.373^{*}$ \\
\hline & Sig. (bilateral) & & .014 \\
\hline & $\mathrm{N}$ & 43 & 43 \\
\hline \multirow{3}{*}{$\begin{array}{l}\text { Ambiente de control } \\
\text { interno }\end{array}$} & Correlación de Pearson & $.373^{*}$ & 1 \\
\hline & Sig. (bilateral) & .014 & \\
\hline & $\mathrm{N}$ & 43 & 43 \\
\hline
\end{tabular}

*. La correlación es significante al nivel 0,05 (bilateral).

Nota: El valor $r$ de Pearson es de 0.373, cuyo valor del Sig, (bilateral) de 0.014, que se encuentra por debajo del 0.05 requerido, por lo cual se objeta la Ho y se acepta la Ha además esta correlación es positiva débil, por lo que se puede afirmar con un $95 \%$ de confianza, que el ámbito de estudio entre el ambiente del control interno y las contrataciones inferiores a 8 UIT en la UGEL Vilcas Huamán, Ayacucho 2021 existe una relación positiva débil $(\mathrm{r}=0.373)$.

\section{Tabla 3}

Pruebas de correlación de la dimensión evaluación de riesgo y la variable de las contrataciones inferiores a 8 UIT en la UGEL Vilcas Huamán, Ayacucho 2021.

\begin{tabular}{llccc}
\hline & Detalle & & $\begin{array}{c}\text { Puntaje total de la } \\
\text { Contrataciones } \\
\text { inferiores a 8 UIT }\end{array}$ & $\begin{array}{c}\text { Evaluación de } \\
\text { riesgo }\end{array}$ \\
\hline $\begin{array}{l}\text { Puntaje total de la } \\
\text { Contrataciones } \\
\text { inferiores a 8 UIT }\end{array}$ & $\begin{array}{l}\text { Correlación } \\
\text { Pearson } \\
\text { Sig. (bilateral) }\end{array}$ & $\mathrm{de}$ & 1 & $.413^{* *}$ \\
\hline & $\mathrm{N}$ & & 43 & .006 \\
Evaluación de riesgo & $\begin{array}{l}\text { Correlación } \\
\text { Pearson }\end{array}$ & $\mathrm{de}$ & $.413^{* *}$ & 43 \\
\hline & $\begin{array}{l}\text { Sig. (bilateral) } \\
\mathrm{N}\end{array}$ & .006 & 1 \\
\hline
\end{tabular}

**. La correlación es significativa al nivel 0,01 (bilateral).

Nota: El valor $\mathrm{r}$ de Pearson es de 0.413, cuyo valor del Sig, (bilateral) de 0.006, que se encuentra por debajo del 0.01 requerido, por lo cual se contradice la Ho y se acepta la Ha además esta correlación es positiva media, por lo que se puede afirmar con un $99 \%$ de confianza, que el ámbito de estudio entre Gestión de riesgo y las contrataciones inferiores 
a 8 UIT en la UGEL Vilcas Huamán, Ayacucho 2021 existe una relación positiva media $(\mathrm{r}=0.413)$.

\section{Tabla 4}

Pruebas de correlación de la dimensión actividad de control y la variable de las contrataciones inferiores a 8 UIT en la UGEL Vilcas Huamán, Ayacucho 2021

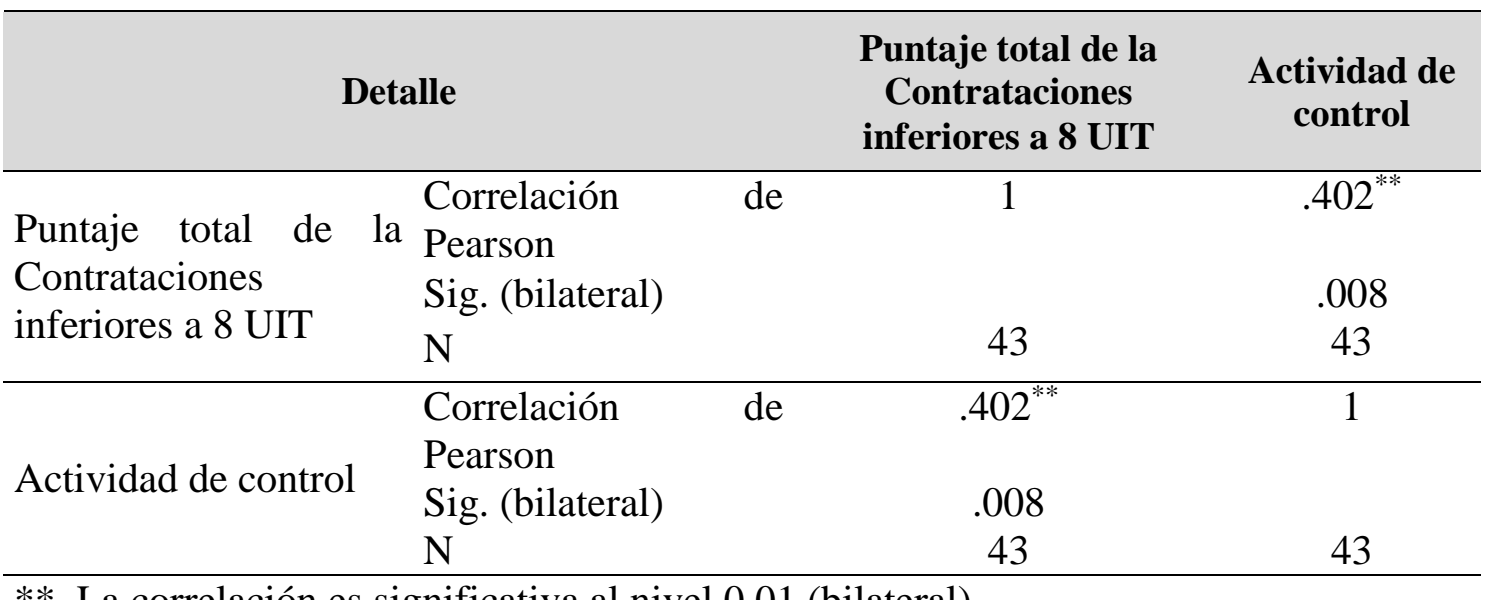

**. La correlación es significativa al nivel 0,01 (bilateral).

Nota: El valor $\mathrm{r}$ de Pearson es de 0.402, cuyo valor del Sig, (bilateral) de 0.008, que se encuentra por debajo del 0.01 requerido, por lo cual se impugna la Ho y se acepta la Ha además esta correlación es positiva media, por lo que se puede afirmar con un $99 \%$ de confianza, que el ámbito de estudio entre Actividades de control y las contrataciones inferiores a 8 UIT en la UGEL Vilcas Huamán, Ayacucho 2021 hay una relación positiva media $(\mathrm{r}=0.402)$.

\section{Tabla 5}

Pruebas de correlación de la dimensión información y Comunicación y variable de las contrataciones inferiores a 8 UIT en la UGEL Vilcas Huamán, Ayacucho 2021.

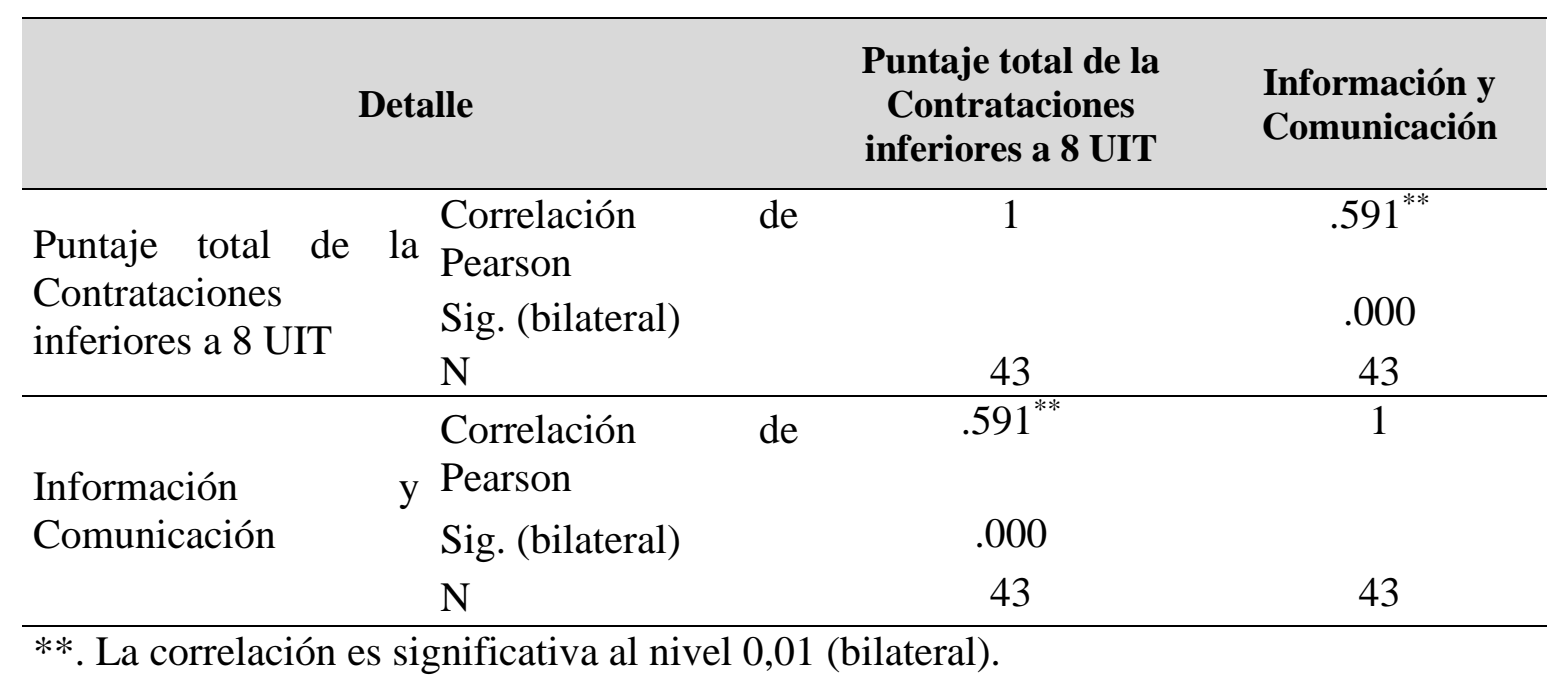


Nota: El valor $\mathrm{r}$ de Pearson es de 0.591, cuyo valor del Sig, (bilateral) de 0.000, que se encuentra por debajo del 0.01 requerido, por lo cual se objeta la Ho y se acepta la Ha además esta correlación es positiva considerable, por lo que se puede afirmar con un $99 \%$ de confianza, que el ámbito de estudio entre Información y comunicación, y las contrataciones inferiores a 8 UIT en la UGEL Vilcas Huamán, Ayacucho 2021 hay una relación positiva considerable $(\mathrm{r}=0.591)$.

\section{Tabla 6}

Pruebas de correlación de la dimensión supervisión y la variable de las contrataciones inferiores a 8 UIT en la UGEL Vilcas Huamán, Ayacucho 2021.

\begin{tabular}{llccc}
\hline & Detalle & & $\begin{array}{c}\text { Puntaje total de la } \\
\text { Contrataciones } \\
\text { inferiores a 8 UIT }\end{array}$ & Supervisión \\
\hline $\begin{array}{l}\text { Puntaje total } \\
\text { Contrataciones } \\
\text { inferiores a 8 UIT }\end{array}$ & $\begin{array}{l}\text { Correlación } \\
\text { Searson }\end{array}$ & de & 1 & $.517^{* *}$ \\
\hline & Sig. (bilateral) & & & .000 \\
Supervisión & Correlación & de & $.517^{* *}$ & 43 \\
& Pearson & & & 1 \\
& Sig. (bilateral) & & .000 & \\
\hline
\end{tabular}

**. La correlación es significativa al nivel 0,01 (bilateral).

Nota: El valor $\mathrm{r}$ de Pearson es de 0.517, cuyo valor del Sig, (bilateral) de 0.000, que se encuentra por debajo del 0.01 requerido, por lo cual se rechaza la Ho y se acepta la Ha además esta correlación es positiva considerable, por lo que se puede afirmar con un $99 \%$ de confianza, que el ámbito de estudio entre Supervisión, y las contrataciones inferiores a 8 UIT en la UGEL Vilcas Huamán, Ayacucho 2021 hay una relación positiva considerable $(\mathrm{r}=0.517)$.

\section{Discusión}

La tabla 1, se aprecia la correlación entre la variable (Control Interno) y la variable (Contrataciones inferiores a 8 UIT) de acuerdo a los participantes y con una significancia (bilateral) obtenido de cero 0,000 lo que confrontado con el parámetro de 0,01 (que representa el 1\% de error y el $99 \%$ de confianza en la determinación de los resultados de acuerdo a la inferencia estadística de la presente investigación) y al ser menor que el parámetro 0.01 nos lleva a objetar la hipótesis general nula (Ho), lo cual nos permite aceptar la hipótesis general positiva (Ha) lo cual menciona si existe relación entre la variable Control Interno y Contrataciones inferiores a 8 UIT (Unidades Impositiva 
Tributaria) en la UGEL (Unidad de Gestión Educativa local) Vilcas Huamán, Ayacucho 2021, en un nivel considerable, se comprueba por Hernández Zarate (2021) quien determino en su estudio que el control interno se relaciona con las compras directas lo cual encontró el resultado de Rho de spearman en 0,655 con un probabilidad de 0.000. Asimismo, García Figueroa (2020) en su investigación determinó que "El control interno y las contrataciones se entrelazan modestamente $(\mathrm{Rho}=0,542)$. De la misma manera Ríos Ramírez (2018) concluyó “el control interno que efectúa el Proyecto, tiene efecto en las compras hasta las 8 UIT", con un coeficiente " $r "=0,764$ señalando que la incidencia entre las variables es efectiva. Igualmente, Maita Casachahua (2018) dijo que control interno incide enormemente en el desempeño institucional, debido a la correlación que existe con los principios del control interno. De la misma forma Albán y Poma (2018) dijeron que al no implementar el control interno afectan la gestión institucional y la probidad de las entidades a nivel nacional, regional y local.

De acuerdo al análisis de los diferentes autores que abordan el tema de control interno y contrataciones menores a 8 UIT, se advierte que el control interno en las entidades públicas presentes en el estado peruano y en otras regiones de América Latina, tienen un impacto positivo en las compras de bienes y servicios para las entidades públicas, entendiéndose que la implementación del control es beneficio para la gestión administrativa.

La tabla 2, se advierte la correlación entre la dimensión (Ambiente de Control) y la variable (Contrataciones inferiores a 8 UIT). Con sig. (Bilateral) obtenido 0.014 lo que, confrontado con el parámetro de 0,05 , y al ser inferior nos lleva a refutar la Ho, aceptando la Ha sobre que, si existe una correlación entre el ambiente del control interno y las contrataciones inferiores a 8 UIT en la UGEL Vilcas Huamán, Ayacucho 2021, a un nivel positivo débil, hecho que es demostrado por Hernández Zarate (2021) quien concluyó que el ambiente de control se relaciona con las compras directas (Rho 0,241 y p-valor 0,038). También García Figueroa (2020) señaló que "el ambiente de control y las contrataciones mínimas se relacionan reservadamente $(\mathrm{Rho}=0,522)$ ”. Asimismo, la Contraloría General de la República (CGR, 2014) y Organización Latinoamericana y del Caribe de Entidades Fiscalizadoras Superiores (OLACEFS, 2015) inciden que el ambiente de control es el pilar para los valores éticos en la organización, manifestación que concuerda con Estupiñan Gaitan (2015) quien dijo que los valores éticos deben ser cumplidos por los 
servidores de la organización durante el tiempo que se encuentre. De tal forma se desprende que el ambiente de control cumple un rol muy importante en la organización ya que establece que todo servidor del estado debe cumplir con los valores de la moral y la integridad para obtener una gestión limpia y transparente.

La Tabla 3, se observa la correlación entre la dimensión (Gestión de Riesgo) y la variable (Contrataciones inferiores a 8 UIT). Con sig. (Bilateral) obtenido 0.006 lo que confrontado con el parámetro de 0,01 y al ser inferior nos lleva a objetar la Ho, aceptando la Ha sobre que, si existe una correlación entre Gestión de riesgo y las contrataciones inferiores a 8 UIT en la UGEL Vilcas Huamán, Ayacucho 2021, siendo positiva media, el presente resultado se comprueba por Hernández Zarate (2021) quien estableció que la valoración de riesgo se relaciona con las compras directas (Rho 0,584 y p-valor 0,000). También dichos resultados concuerdan con García Figueroa (2020) quien expresó que "la evaluación de riesgo y las compras bajas se relacionan moderadamente con un $(\mathrm{Rho}=0$, 466)". Sin embargo, Ruiz et. al., (2020) expresaron que, en la evaluación de riesgo, no se realiza la valoración de riesgo, donde se requiere afinar la ejecución de los métodos de contratación de bienes y servicios. Igualmente, Fazekas \& Wachs (2020) manifestaron que el cambio político tiene un efecto significativo en las relaciones corruptas: los compradores con alto riesgo de corrupción con vecindarios dispersos reconfiguran sus relaciones contractuales aproximadamente entre un $20 \%$ y un $40 \%$ más ampliamente que otros compradores a lo largo de los años con rotación del gobierno". De igual forma Ordoñez et. al., (2019) concluyeron que el control interno detecta y corrige errores, de esta manera la información de la organización tendrá más razonabilidad, integridad y fiabilidad. Por lo tanto, se discute que la gestión de riesgo debe ser fortalecida con la finalidad de identificar los riesgos de manera oportuna y ser corregidos en su momento oportuno.

La Tabla 4, se advierte la correlación entre la dimensión (Actividades de control) y la variable (Contrataciones inferiores a 8 UIT). Con sig. (Bilateral) obtenido 0,008 lo que confrontado con el parámetro de 0,01 y al ser mínima nos lleva a contradecir la Ho, aceptando la Ha sobre que, si existe correlación entre Actividades de control y las contrataciones inferiores a 8 UIT en la UGEL Vilcas Huamán, Ayacucho 2021, siendo un nivel medio, se comprueba lo hallado por Hernández Zarate (2021) quien en su estudio comprobó que la actividad de control se relaciona con las compras directas (Rho 0,437 y 
p-valor 0,000). Resultados que también concuerdan con García Figueroa (2020) donde estableció que "las actividades de control se correlacionan con las compras inferiores, (Rho 0,437 y p-valor 0,000). Sin embargo, Ruiz et. at., (2020) expresaron que Actividad de control, es urgente realizar acciones que mejoren el control interno. Del análisis se advierte que las actividades de control es un procedimiento que apoya a las contrataciones directas inferiores a 8 UIT.

La Tabla 5, se indica la correlación entre la dimensión (Información y comunicación) y la variable (Contrataciones inferiores a 8 UIT). Con sig. (Bilateral) obtenido 0,000 lo que confrontado con el parámetro de 0,01 y al ser inferior nos lleva a rechazar la Ho, aceptando la Ha sobre que si existe correlación entre información y comunicación, y las contrataciones inferiores a 8 UIT en la UGEL Vilcas Huamán, Ayacucho 2021 siendo positivo considerable, se comprueba lo hallado por Hernández Zarate (2021) quien en el referido estudio finalizó que la información y comunicación se relaciona con las compras directas (Rho 0,357 y p-valor 0,002). De igual forma García Figueroa (2020) expresa que "La información y comunicación y las contrataciones menores se relacionan prudentemente (Rho=0,565)". Asimismo, Ruiz et. al., (2020) dijeron que Información y comunicación, realizar tácticas enlazadas en el área de logística. De igual forma la CGR (2014) y OLACEFS (2015) establecieron que la comunicación e información, es necesario en la institución llevar a cabo el logro de sus objetivos, las cuales pueden ser interna y externa ya que cada una de ellas cumplen un rol importante dentro y fuera de la entidad, a la vez dice que una comunicación eficaz que vaya en todas direcciones de la organización. Del análisis realizado a los hechos descritos por los autores, se advierte que la comunicación y la información en una entidad, es de mucha importancia porque apoya a que las personas se comuniquen dentro y fuera de la entidad.

La Tabla 6, se expone la correlación entre la dimensión (Supervisión) y la variable (Contrataciones inferiores a 8 UIT). Con sig. (Bilateral) obtenido 0,000 lo que confrontado con el parámetro de 0,01 y al ser pequeño nos lleva a desaprobar la Ho, aceptando la Ha sobre que, si existe correlación entre supervisión y las contrataciones inferiores a 8 UIT en la UGEL Vilcas Huamán, Ayacucho 2021 siendo positivo considerable, se acredita lo descubierto por Hernández Zarate (2021) quien en su averiguación estableció que la supervisión se relaciona con las compras directas (Rho 0,330 y p-valor 0,004). Asimismo, García Figueroa (2020) establece que "la supervisión 
y las compras inferiores se relacionan prudentemente con un $(\mathrm{Rho}=0,582)$ ". Al mismo tiempo Ruiz et. al., (2020) expresaron que la Supervisión, se debe fortalecer un mecanismo de prevención y seguimiento de resultados para establecer planes de mejora y educir los riesgos en el área de logística. A su vez la CGR (2014) y OLACEFS (2015) manifestaron que lado la supervisión tiene como función evaluar constantemente el control interno en las principales deficiencias a fin de ser mejorados oportunamente y debe ser continua y periódica.

\section{CONCLUSIÓN O CONSIDERACIONES FINALES}

1. Se concluye que la relación entre Control Interno y Contrataciones inferiores a 8 UIT en la UGEL Vilcas Huamán, Ayacucho 2021, no solo existe si no que es positiva: Demostrando que el control interno es muy importante en las contrataciones inferiores a 8 UIT, es positiva considerable $(r=0.565)$.

2. Se determinó que la incidencia de relación entre el ambiente del control interno y las contrataciones inferiores a 8 UIT en la UGEL Vilcas Huamán, Ayacucho 2021, siendo positiva débil $(\mathrm{r}=0.373)$.

3. Se concluye que la dimensión Gestión de Riesgo se relaciona con la variable Contrataciones inferiores a 8 UIT en la UGEL Vilcas Huamán, Ayacucho 2021, positiva considerable $(\mathrm{r}=0,584)$.

4. Se finalizó que la dimensión Actividades de control se relaciona con la variable Contrataciones inferiores a 8 UIT en la UGEL Vilcas Huamán, Ayacucho 2021. siendo positiva media $(\mathrm{r}=0.402)$.

5. Se culminó que la dimensión Información y comunicación se relaciona con la variable contrataciones inferiores a 8 UIT en la UGEL Vilcas Huamán, Ayacucho 2021. siendo positiva considerable $(\mathrm{r}=0.591)$.

6. Se concluyó que la dimensión Supervisión se relaciona con la variable Contrataciones inferiores a 8 UIT en la UGEL Vilcas Huamán, Ayacucho 2021. siendo positiva considerable $(\mathrm{r}=0.517$ 


\section{LISTA DE REFERENCIAS}

Albán Sáenz , J. M., \& Poma Vargas , A. E. (31 de Octubre de 2018). Problematica del sistema de control interno para erradicar la corrupción en el estado peruano. Yachaq, 01(02), 73-96. Recuperado el 11 de Setiembre de 2021, de https://revista.uct.edu.pe/index.php/YACHAQ/article/view/70/47

Apolo Ordoñez, G. M., Narváez Zurita, C. I., \& Erazo Álvarez, J. C. (2019). El Control interno como herramienta de apoyo a la gestión financiera del Gobierno Autónomo Descentralizado Municipal Zaruma. CIENCIAMATRIA, 551-578. Recuperado el 2 de Octubre de 2021, de https://www.cienciamatriarevista.org.ve/index.php/cm/article/view/280/327

Arribasplata Sánchez, D. (2018). Actividades de control gerencial y su contribución en la ejecución de contrataciones iguales o inferiores a 8 UIT en la Sede Central del Gobierno Regional Cajamarca, año 2016. Cajamarca: Universidad Nacional de Cajamarca. Recuperado el 5 de Setiembre de 2021, de https://repositorio.unc.edu.pe/bitstream/handle/UNC/2350/Actividades\%20de\%2 0control\%20gerencial\%20y\%20su\%20contribuci\%c3\%b3n\%20en\%20la\%20eje cuci\%c3\%b3n\%20de $\% 20$ contrataciones $\% 20$ iguales $\% 20 \mathrm{o} \% 20 \mathrm{in}$. pdf?sequence= $1 \&$ isAllowed=y

Azalgara Bedoya, M. J. (2019). Analisis de las Contrataciones menores o iguales a las 8 UIT al margen de la Ley de Contrataciones con el Estado: Ponderación entre el dinamismo de la Contratación Pública y el Control de Contrataciones Estatales. Arequipa: Universidad Catolica San Pablo. Recuperado el 5 de Setiembre de 2021 ,

http://repositorio.ucsp.edu.pe/bitstream/UCSP/16029/4/AZALGARA_BEDOYA _MAR_CON.pdf

Carrasco Diaz, S. (2017). Metodologia de la investigación cientifica. En S. Carrasco Diaz, Metodologia de la investigación cientifica (pág. 476). Lima, Lima, Perú: San Marcos E.I.R.L. Recuperado el 24 de Setiembre de 2021

CGR. (Agosto de 2014). Google. Recuperado el 19 de Setiembre de 2021, de https://apps.contraloria.gob.pe/wcm/control_interno/documentos/Publicaciones/ Marco_Conceptual_Control_Interno_CGR.pdf 
Estupiñán Gaitán, R. (2015). Control interno y fraudes. Bogota - Colombia: Ecoe Ediciones Ltda. Recuperado el 19 de Setiembre de 2021, de https://books.google.com.pe/books?id=qcO4DQAAQBAJ\&printsec=frontcover $\& d q=$ REVISTA+CIENTIFICA+Evaluaci\%C3\%B3n+de+riesgos+control+intern o\&hl=es\&sa=X\&ved=2ahUKEwjnnLWtuIzzAhXbr5UCHYUwC1AQ6wF6BA gLEAE\# $\mathrm{v}=$ onepage $\& \mathrm{q} \& \mathrm{f}=$ false

García Figueroa, B. (2020). Control interno y compras públicas menores a 8 UIT en la Municipalidad Distrital de Pariahuanca, Ancash, 2019. Lima-Perú: Universidad Cesar Vajello. Recuperado el 25 de Noviembre de 2021, de https://repositorio.ucv.edu.pe/bitstream/handle/20.500.12692/44703/Garc\%c3\%a da_FBZ-SD.pdf?sequence $=1 \&$ isAllowed $=y$

Hernández Sampieri, R., Férnandez Collado, C., \& Baptista Lucio, P. (2014). Metodologia de la investigación. En R. Hernández Sampieri, C. Férnandez Collado, \& P. Baptista Lucio, Metodologia de la investigación (pág. 589). Mexico, Mexico, Mexico: McGRAW-HILL / Interamericana Editores S.A. de C.V. Recuperado el 25 de Setiembre de 2021

Hernández Zarate, G. (2021). Control interno y compras directas menores a 8 UIT en el Programa Qali Warma, Santiago de Surco, 2020. Lima - Perú: Universidad Cesar Vallejo. Recuperado el 21 de Noviembre de 2021, de https://repositorio.ucv.edu.pe/bitstream/handle/20.500.12692/58132/Hernandez ZGR-SD.pdf? sequence $=1 \&$ is Allowed $=\mathrm{y}$

Maita Casachahua, M. L. (2018). Implementaciòn del control interno y su influecia en el desempeño de la instituciòn administradora de fondo de aseguramiento en salud del ejercito del Perù. Universidad Nacional Federico Villa Real, Lima. Lima Perù: Universidad Nacional Federico Villareal. Recuperado el 20 de Setiembre de 2021 , http://repositorio.unfv.edu.pe/bitstream/handle/UNFV/2515/Maita\%20Casachah ua\%20Maribel\%20Luisa\%20-Maestria.pdf?sequence=1\&isAllowed=y

Miaja Fol, M. (2019). Presente y futuro del Control Interno en las Administraciones Públicas. Auditoria y gestion de los fondos públicos, 85-98. Recuperado el 1 de Octubre de 2021, de https://asocex.es/wp-content/uploads/2019/12/RevistaAuditoria-Publica-n\%C2\%BA-74.-pag-85-a-98.pdf 
Mihály Fazekas, \& Johannes Wachs. (2020). Corruption and the Network Structure of Public Contracting Markets across Government Change. Politics and Governance, 8(2). Recuperado el 30 de Diciembre de 2021, de https://www.cogitatiopress.com/politicsandgovernance/article/view/2707

Montiel Sandoval, M. E., Montiel Sandoval, C. C., \& Montiel Sandoval, Ó. A. (2017).

La implementación del Control Interno fortalece la gobernabilidad en las alcaldias municipales en Colombia. Administración\&Desarrollo(47), 97-117. Recuperado el 5 de Setiembre de 2021, de file:///C:/Users/cpcma/Downloads/DialnetLaImplementacionDelControlInternoFortaleceLaGobern-6403441.pdf

OLACEFS. (Noviembre de 2015). Goolge. Recuperado el 20 de Setiembre de 2021, de https://www.olacefs.com/wp-content/uploads/2016/03/15.pdf

PROETICA. (2020). índice de la percepcion de la corrupción 2019. Transparency International, 29. Recuperado el 1 de Octubre de 2021, de https://www.proetica.org.pe/wp-content/uploads/2020/01/CPI2019_Report_ESWEB.pdf

Ruiz Correa, S., Delgado Bardales, J. M., Ruiz Correa, J., Olivas Salazar, H., \& Enriquez Calderon, R. A. (2020). Control interno para mejorar las contrataciones del área de logistica, Unidad de Gestión Educativa Local San Martin 2020. Ciencia Latina Revista multidiciplinar, 4(2), 936-954. Recuperado el 11 de Setiembre de 2021, de https://ciencialatina.org/index.php/cienciala/article/view/132/151

Valderrama Mendoza, S. (2019). Pasos para elaborar proyectos de investigacion cientifica, Cuantitativa, Cualitativa y Mixta. En S. V. Mendoza, Pasos para elaborar proyectos de investigacion cientifica, Cuantitativa, Cualitativa y Mixta (Decima ed., pág. 495). Lima, Lima, Perú: San Marcos E.I.R.L. Recuperado el 24 de Setiembre de 2021 